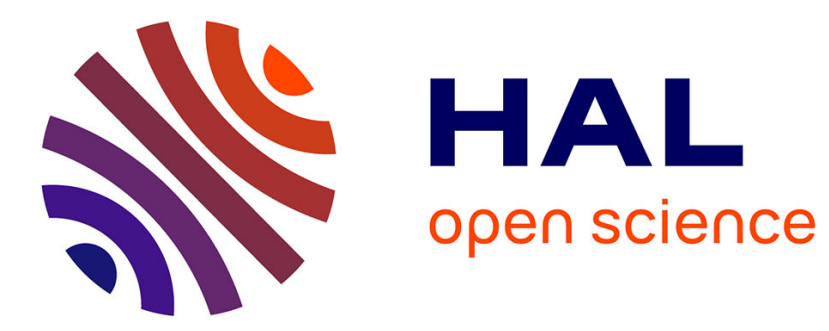

\title{
Viscoelastic properties of confined molecular layers
}

\author{
Alois Würger
}

\section{To cite this version:}

Alois Würger. Viscoelastic properties of confined molecular layers. Journal of Physics: Condensed Matter, 2011, 23 (50), pp.505103. 10.1088/0953-8984/23/50/505103 . hal-00641816

\section{HAL Id: hal-00641816 https://hal.science/hal-00641816}

Submitted on 16 Nov 2011

HAL is a multi-disciplinary open access archive for the deposit and dissemination of scientific research documents, whether they are published or not. The documents may come from teaching and research institutions in France or abroad, or from public or private research centers.
L'archive ouverte pluridisciplinaire HAL, est destinée au dépôt et à la diffusion de documents scientifiques de niveau recherche, publiés ou non, émanant des établissements d'enseignement et de recherche français ou étrangers, des laboratoires publics ou privés. 


\title{
Viscoelastic properties of confined molecular layers
}

\author{
Alois Würger \\ Laboratoire Ondes et Matière d'Aquitaine, Université de Bordeaux \& CNRS, \\ 351 cours de la Libération, 33405 Talence, France
}

\begin{abstract}
We study the viscoelastic properties of a film of $n$ layers of spherical molecules confined between two walls. We find that the dynamic response arises from two competing contributions: the effective stiffness of $n+1$ springs in series and softening due to strain fluctuations. In particular, the latter are of the origin of the oscillatory behavior of stiffness and damping coefficient. Dissipation is strongest at the minima of the stiffness; the inverse behavior may occur for a modulated relaxation time. As a corollary we show that confined molecular layers cannot be described as Maxwell fluids.
\end{abstract}

\section{INTRODUCTION}

Molecular liquids confined at a nanometer scale undergo striking structural changes. Their viscoelastic properties differ significantly from those of the bulk and cannot be understood in terms of continuum fluid mechanics [1]. Surface-force apparatus (SFA) and atomic-force microscopy (AFM) studies on molecular liquids report oscillatory solvation forces and give evidence for the layered structure of the film [2-6], in agreement with numerical simulations of the molecular dynamics [7]. When reducing its thickness $L$ one observes a sequence of solid-like states, which are separated by rather unstable configurations related to the expulsion of a molecular layer. These alternating states explain the periodic modulation of the elastic properties: The maximum stiffness occurs for the commensurate case with the film thickness corresponding to an integer number of molecular layers [8-19].

A more complex picture, however, emerges regarding the modulation of the viscous forces exerted by a confined film on a vibrating AFM tip. Experimental findings of different groups disagree on whether or not the dissipative response shows an oscillatory behavior as the film thickness is reduced. Several studies on water [10-12], octamethyltetracyclosiloxane (OMTCS) [13, 14], and dodecanol [17] reported a modulation of viscoelastic response, with a period corresponding to the molecular size; the maxima of stiffness and dissipation may coincide, or be shifted by half a period. These discrepancies have been related to a cross-over from liquid to solid behavior due to "jamming" [13], or could partly be due to the complex data analysis $[15,16]$. Very recent experiments confirm the oscillations of the damping coefficient: For a small AMF tip, its maxima coincide with minimum stiffness [18]; a more complex situation occurs for a larger tip with a rough surface [19].

In the present Letter we derive the viscoelastic linear response function. The free energy of $n$ layers is calculated in harmonic approximation for the molecular interactions; local fluctuations are included in a grandcanonical approach with variable $n$. The dynamic stiffness and damping coefficient are obtained from Kubo's complex response function in relaxation time approximation. Configurational fluctuations in the intermediate states are shown to play a central role for the oscillatory

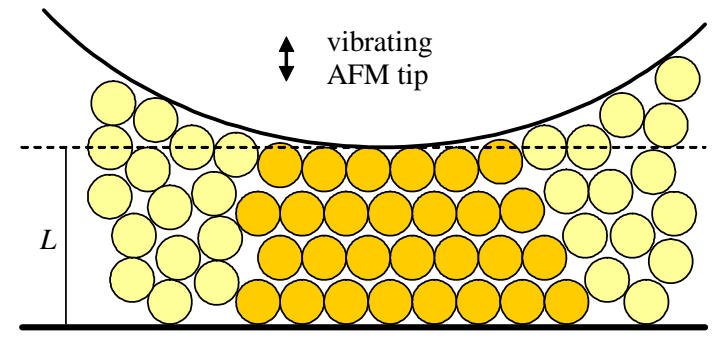

FIG. 1: Schematic view of the confinement-induced ordering of a liquid film. The ordered domain is indicated by darker beads; its area $A \approx a R$ is given by the curvature radius $R$ of the AFM tip and the molecular radius $a$.

behavior observed in experiments.

\section{DYNAMIC RESPONSE}

The thermodynamic potential $\Omega$ of a confined molecular liquid depends on the film thickness $L$. For thick films the solvation force

$$
F=-d \Omega / d L
$$

is constant and simply related to the hydrostatic pressure. Structuration effects in nanoscale films, however, lead to an oscillatory behavior of both the force $F$ and the static stiffness

$$
K_{0}=\frac{d^{2} \Omega}{d L^{2}}
$$

Viscoelastic properties are obtained from the dynamic response function

$$
\phi\left(t-t^{\prime}\right)=\left\langle\left\{F(t), F\left(t^{\prime}\right)\right\}\right\rangle,
$$

which is given by the Poisson brackets of the fluctuating part of the solvation force [20]. The time dependent force $F(t)$ is realized by vibrating an AFM tip at frequency $\omega$ and amplitude $A$, as illustrated in Fig. 1 . The linear response approximation is justified if $A$ is significantly smaller than the molecular size. The real parts of the Fourier transform $\phi(\omega)=\phi^{\prime}(\omega)+\phi^{\prime \prime}(\omega)$ gives the 
dynamic contribution to the film stiffness

$$
K(\omega)=K_{0}+\phi^{\prime}(\omega)
$$

whereas the imaginary part is related to the damping coefficient,

$$
\gamma=\frac{\phi^{\prime \prime}(\omega)}{\omega}
$$

Experimentally these quantities are extracted from the measured frequency shift and the attenuation of the vibration of the AFM tip.

In the following we evaluate the grand potential $\Omega$ in terms of the $n$-layer free energy in harmonic approximation. Since we are mainly interested in structuration effects, we consider the response function $\phi$ due to local fluctuations of the number of layers $n$.

\section{HARMONIC APPROXIMATION}

As suggested by the force experiments and confirmed by numerical work [7], the molecules in a confined film arrange in a regular array, as illustrated in Fig. 1. We start from a close packed structure [21] of $n$ layers parallel to the confining walls; other symmetries may occur [22-25] but would not change the essential aspects. The potential energy of the ordered domain then reads

$$
E=\sum_{\langle i, j\rangle} v\left(r_{i j}\right)+\sum_{\langle i, w\rangle} v_{0}\left(r_{i w}\right)
$$

where the first sum runs over 12 nearest neighbors. The second term accounts for the interaction of the upper and lower layers with the molecules of the confining walls. Expanding the molecular Lennard-Jones potentials to quadratic order in the distance, we have $v(r)=\frac{1}{2} k(r-a)^{2}$. A similar expression with a constant $k_{0}$ arises for the interaction $v_{0}$ with the confining walls.

Elastic theory for an infinite system usually relies on the strain tensor $\varepsilon_{\alpha \beta}$ which is defined by deformation of the distance vector $\mathbf{r}_{i j}$ with respect to that of the perfect lattice [21]. In the present case the strain arises from the boundary conditions imposed by the confining walls. The vertical mismatch with respect to the thickness of $n$ unstrained layers reads as

$$
\ell_{n}=L-n \tilde{a}, \quad \tilde{a}=\sqrt{\frac{2}{3}} a,
$$

where $\tilde{a}$ is the effective spacing in a cubic crystal and where we have discarded an irrelevant constant due to the boundary layers. The mismatch is related to the diagonal strain element through $\ell_{n}=\varepsilon_{x x} n \tilde{a}$.

Expanding (6) in powers of the vertical deviations $\hat{x}_{i}$ from the equilibrium position of molecule $i$, we obtain

$$
E=\frac{1}{2} \sum_{\langle i, j\rangle} k_{i j}\left(\hat{x}_{i}-\hat{x}_{j}\right)^{2} .
$$

The $k_{i j}$ are finite for nearest neighbors only and take the values $\frac{2}{3} k$ and $\frac{2}{3} k_{0}$. As a consequence, the canonical partition function of a $n$-layer configuration reduces to a Gaussian form. Summing over the 3 neighbors in each adjacent layer and integrating over the $\hat{x}_{i}$, one obtains the canonical free energy

$$
\Omega_{n} / N=\frac{k_{n}}{2} \ell_{n}^{2}+\frac{1}{2} k_{B} T \ln \tilde{n} .
$$

where $N$ is the number of molecules per layer.

The first term is the elastic energy, which depends on the square of the vertical mismatch $\ell_{n}$ and the effective stiffness

$$
k_{n}=\frac{2 k}{\tilde{n}}, \quad \tilde{n}=(n-1)+\frac{2 k}{k_{0}} .
$$

The physical origin of $k_{n}$ becomes obvious when writing $2 / k_{n}=(n-1) / k+2 / k_{0}$ : For $n$ molecular layers, there are $n-1$ inter-layer couplings and the interaction of the outer layers with the solid boundaries. The case $n=1$ describes a single layer coupled to both confining walls. For a few layers, the overall stiffness $k_{n}$ is determined by the softer of the springs. We use $k_{0}=k$ throughout this paper. Yet note that a strong coupling to the confining walls would tightly bind the outer molecular layers to the surfaces, whereas the opposite case $\left(k_{0} \ll k\right)$ leads to a more complex alteration of the elastic response.

In Fig. 2 we plot $\Omega_{n}$ as a function of the spacing $L$ for several values of $n$. The elastic energy results in a parabola centered at $\ell_{n}=0$, with a constant given by the second term in Eq. (8). If the vertical mismatch is much smaller than the molecular size, $\left|\ell_{n}\right| \ll \tilde{a}$, adding or subtracting a layer would cost a considerable amount of energy; thus the $n$-layer configuration is stable. A different situation arises if the values of the free energy corresponding to $n$ and $n+1$ are comparable. In terms of the mismatch this means $\ell_{n} \approx-\ell_{n+1}$. Then there are local fluctuations of small groups of particles between configurations $n$ and $n+1$, as illustrated in Fig. 2 for $n=3$. The resulting grandcanonical potential

$$
\Omega=-N k_{B} T \ln \sum_{n} e^{-\Omega_{n} / N k_{B} T}
$$

is minimum for stable configurations and maximum at the intermediate states. This form is obtained under the assumption that the film is drained bead by bead through independent relaxation processes. If, on the contrary, a layer of $N$ molecules was expelled as a whole, one would have to suppress both factors $N$ occurring in (10).

The number of molecules in one ordered layer is given by the ratio $N=A / A_{1}$ of the domain size $A$ and the area per molecule $A_{1}=\tilde{a}^{2} \sqrt{3 / 4}$ [21]. In order to obtain an estimate for the former, we note that the spacing $h$ between tip and solid support varies as $h=L+R-$ $\sqrt{R^{2}-r^{2}}$, where $r$ is the lateral distance from the tip axis. Existence of an ordered state requires that $h-L$ be small as compared to the molcular size; neglecting a 


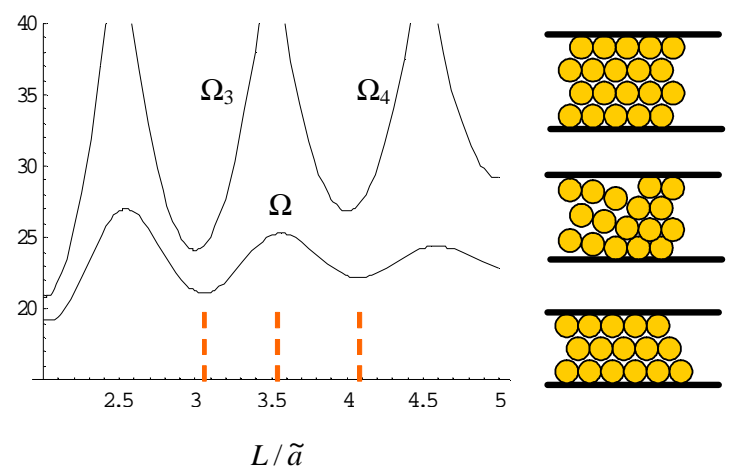

FIG. 2: Grand potential $\Omega$ and canonical excess free energies $\Omega_{n}$ in units of $k_{B} T$. Minima of $\Omega$ correspond to stable ordered configurations where the film thickness $L$ is close to a multiple integer of $\tilde{a}$. We put $k=0.1 \mathrm{~N} / \mathrm{m}, \tilde{a}=0.8 \mathrm{~nm}$, and $N=$ 30 . The intermediate states are highly strained and result in strong fluctuations of the local configuration.

numerical factor we find the domain radius $r_{0} \approx \sqrt{R a}$. Thus the area $A \approx \pi R a$ and the corresponding number of molecules

$$
N \approx \pi R / a
$$

increase linearly with the tip radius, and so does the free energy (8).

When varying the film thickness $L$, the grand potential $\Omega$ oscillates with period $\tilde{a}$, and so do its derivatives. We start with the solvation force (2) exerted by the film on the walls. Taking the derivative, one finds $F$ as the mean of the force in an $n$-layer configuration,

$$
F / N=\left\langle f_{n}\right\rangle, \quad f_{n}=-k_{n} \ell_{n},
$$

where the average over the number of layers reads explicitly

$$
\langle\ldots\rangle=\sum_{n}(\ldots) e^{\left(\Omega-\Omega_{n}\right) / N k_{B} T} .
$$

(Here and in the following, we consider the force per molecule in one layer, $F / N$, which is identical to the disjoining pressure multiplied with the area per molecule.)

From the curve $\Omega$ in Fig. 2 it is clear that its slope $F$ oscillates as a function of $L$, in perfect agreement with various measurements on molecular liquids in the range $n=2 \ldots 7$. The envelope function decreases exponentially, $F \sim e^{-L / \xi}$; for large stiffness the characteristic length $\xi$ is proportional to $k$. The oscillatory solvation force has been reproduced by density-functional theory hardsphere systems [26]. The overall picture is confirmed by the accord of experimental and numerical studies of the drainage kinetics [28-30].

\section{THE ROLE OF FLUCTUATIONS}

At maximum mismatch, two adjacent states of similar free energies $\Omega_{n} \approx \Omega_{n+1}$ are equally probable, and the resulting fluctuations strongly affect the elastic response of the film. The underlying mechanism is best exposed in terms of the stiffness, or static susceptibility (3). From the grand potential one readily calculates

$$
\frac{K_{0}}{N}=\left\langle k_{n}\right\rangle-\frac{\delta f^{2}}{k_{B} T} .
$$

These two contributions are of rather different physical origin. The first one is given the mean stiffness $\left\langle k_{n}\right\rangle$; it decreases monotonously with the inverse film thickness, as is clear from (9). The second one arises from the fluctuations of the solvation force,

$$
\delta f^{2}=\left\langle f_{n}^{2}\right\rangle-\left\langle f_{n}\right\rangle^{2} .
$$

If $n$ layers correspond to optimal filling $\ell_{n} \approx 0$, adding or subtracting a layer requires a significant amount of energy; then the states $n \pm 1$ are hardly occupied, $\delta f^{2}$ is small, and the stiffness is positive. If, on the other hand, the system size $L$ lies between two values of optimal filling, fluctuations $n \rightleftarrows n+1$ are important and soften the elastic response of the confined liquid such that $K_{0}<$ 0 . The curves labeled $\infty$ and 0 in the upper panel of Fig. 3 correspond to $N\left\langle k_{n}\right\rangle$ and $K_{0}$, respectively; they show that the leading terms of $\left\langle k_{n}\right\rangle$ and $\delta f^{2} / k_{B} T$ vary linearly with the inverse film thickness and cancel each other. The oscillating behavior of $K_{0}$ is due to the fluctuations $\delta f^{2}$; the envelope decreases exponentially $\sim e^{-L / \xi}$ with $\xi \approx 2 \tilde{a}$.

So far we have considered the static response of the confined film, where the change of the external control parameter $L$ is much slower than the characteristic time $\tau$ of a configuration change. Dynamic effects are accounted for by the retarded response

$$
\phi\left(t-t^{\prime}\right)=N\left\langle\left\{f_{n}(t), f_{n}\left(t^{\prime}\right)\right\}\right\rangle,
$$

which is given by the Poisson brackets of the fluctuating part of the solvation force [20]. When assuming the fluctuations $\delta f^{2}$ to occur on a single time scale $\tau$, one has for its Fourier transform

$$
\frac{\phi(\omega)}{N}=\frac{\delta f^{2}}{k_{B} T} \frac{i \omega \tau}{1+i \omega \tau} .
$$

The complex susceptibility of a confined film is given by the sum $K_{0}+\phi(\omega)$. Its real part is the frequencydependent stiffness $K=K_{0}+\phi^{\prime}$, which after rearranging the fluctuation parts reads as

$$
\frac{K(\omega)}{N}=\left\langle k_{n}\right\rangle-\frac{\delta f^{2}}{k_{B} T} \frac{1}{1+\omega^{2} \tau^{2}} .
$$

Since at finite frequencies the fluctuation-driven part diminishes, the overall stiffness increases with $\omega$, as illustrated in the upper panel of Fig. 3. The modulation as a function of the film thickness is due to the mean square force $\delta f^{2}$.

The quantity $K(\omega)$ describes the linear response to a periodic variation $A \cos (\omega t)$ of the thickness. In the limit 

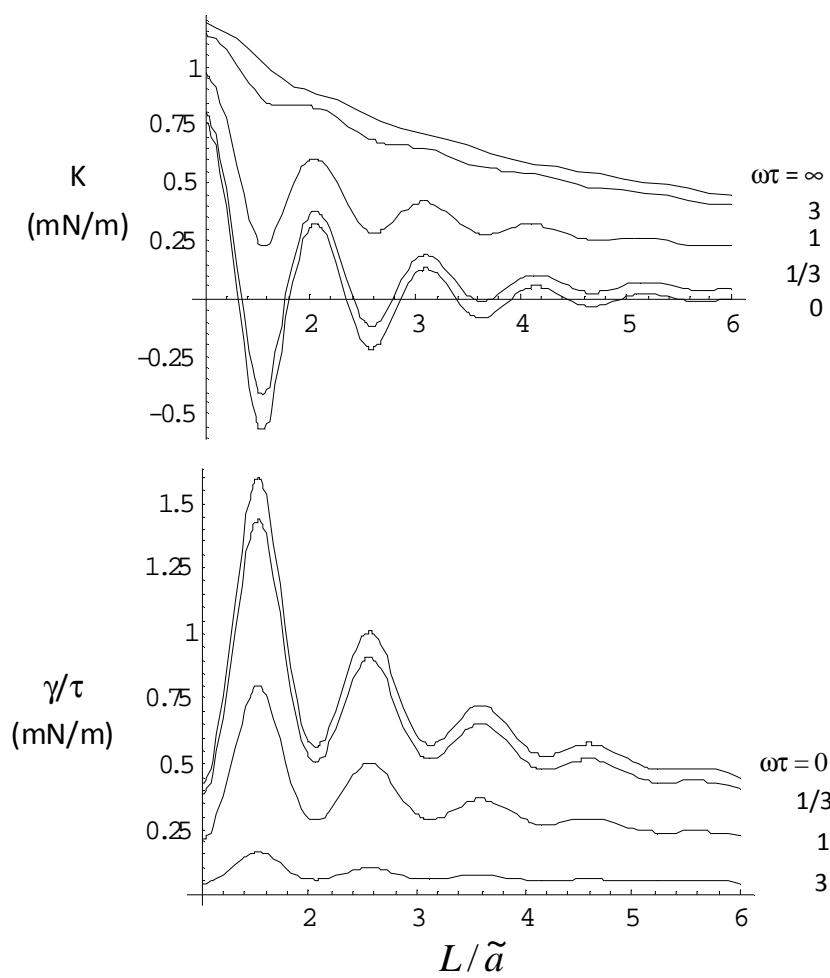

FIG. 3: Dynamic stiffness $K(\omega)$ and reduced damping coefficient $\gamma / \tau$ for different values of $\omega \tau$. Increasing $\omega \tau$ augments the stiffness but reduces its modulation amplitude. The damping coefficient $\gamma / \tau$ is maximum in the static limit. The modulation is entirely due to the force fluctuations of Eq. (13). Note that damping maxima occur at minimum stiffness. Parameters are $k=0.06 \mathrm{~N} / \mathrm{m}, \tilde{a}=0.8 \mathrm{~nm}$, and $N=30$.

of a rapid perturbation $(\omega \tau \gg 1)$, where the duration of one cycle is much shorter than the mean relaxation time $\tau$, the film does not have the time to adjust its configuration. Then the stiffness is given by the quasiinstantaneous response, that is, by the mean spring constant $\left\langle k_{n}\right\rangle$. In the case $\omega=0$ the external perturbation is slow, allowing the system to fully relax; the fluctuations reduce the stiffness to its static value (12). Note that the high-frequency response is always positive, whereas at low $\omega$ it may take either sign. The curves for small $\omega \tau$ agree rather well with experiment [9-14].

The imaginary part of (15) gives the confinementinduced damping coefficient $\gamma=\phi^{\prime \prime} / \omega$,

$$
\frac{\gamma(\omega)}{N}=\frac{\delta f^{2}}{k_{B} T} \frac{\tau}{1+\omega^{2} \tau^{2}}
$$

Assuming a constant relaxation time one finds that $\gamma$ decreases with increasing frequency. The modulation as a function of $L$ shown in the lower panel of Fig. 3, is due to the force fluctuations; damping minima occur at optimal filling where $\delta f^{2}$ is small, and the maxima in the intermediate states.

\section{SHEAR RESPONSE FUNCTION}

The present paper deals with motion perpendicular to the film. Formally our results apply equally well to shear motion, where the external driving force is parallel to the solid support. Experimentally this could be realized by horizontal small-amplitude vibrations of the AFM tip of Fig. 1. Here we address several aspects that may lead to important differences in the shear or draining response function.

The molecular ordering in a thin film is anisotropic. As shown in Fig. 1, the vertical confinement imposes a unique mismatch $\ell_{n}$; the corresponding static stress is homogeneous throughout the confined volume. This is quite different in the parallel directions. If the surfaces were structured with the period corresponding to the molecular size, they would pin the first liquid layer and result in an elastic response function similar to (16). Commonly used materials such as silicates and metal coatings, however, provide rather flat surfaces; the molecules move freely along the solid boundaries and thus rapidly relax upon a static shear. In other words, there is no permanent lateral mismatch between the first and last layer. As a consequence, the static shear stiffness vanishes, and the response function consists of the dynamic suseptibility $\phi_{S}$ only. Thus the stiffness reduces to the relaxator contribution

$$
\frac{K_{S}(\omega)}{N}=\frac{\delta f_{S}^{2}}{k_{B} T} \frac{\omega^{2} \tau_{S}^{2}}{1+\omega^{2} \tau_{S}^{2}}
$$

whereas the damping coefficient is similar to (17), albeit with parameters $\delta f_{S}$ and $\tau_{S}$. In particular this results in a linear frequency dependence of the ratio of elastic and viscous forces, $\phi_{S}^{\prime} / \phi_{S}^{\prime \prime}=\omega \tau_{S}$, which is characteristic for the liquid state [27]. For the force fluctuations and the relaxation time one expects a behavior similar to that discussed above for the normal response function.

Many shear studies apply amplitudes well beyond the molecular size, and thus do not satisfy the limitations of the linear-response approximation used in the present work. Similarly, squeezing out successive molecular layers probes the rheology on large scales [28-30], which is not directly related to the small-amplitude vibrations. Like any complex many-body system, molecular films show a broad relaxation spectrum. Each experiment probes a particular motion, and the resulting time constants may be quite different.

\section{DISCUSSION}

The oscillatory behavior of the solvation force is well established experimentally, and has been related to the the number of molecular layers in the film [1-7]. The role of fluctuations is illustrated in Fig. 2; the force $F$ and stiffness $K_{0}$ are readily obtained from the local slope and curvature of the grand potential $\Omega$, according to (1) 


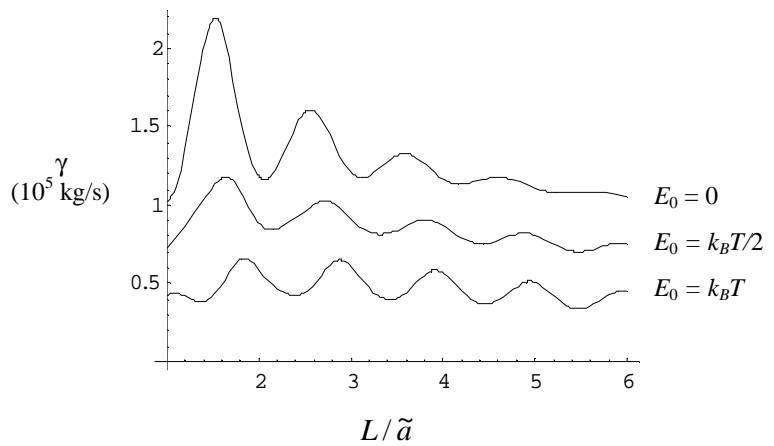

FIG. 4: Damping constant $\gamma$ for a non-uniform relaxation time $\tau=\tau_{0} e^{E_{L} / k_{B} T}$, with a modulated activation energy $E_{L}=E_{0}[1+\cos (2 \pi L / \tilde{a})] \tilde{a} / L$. The curve for $E_{0}=0$ is identical to those of Fig. 3, with damping maxima in the intermediate states. A finite activation energy shifts the oscillations; for $E_{0}>k_{B} T$ the maxima of $\gamma$ occur in the ordered state, in phase with those of the stiffness. For the sake of clarity, we have shifted the vertical scale of the upper curves. Parameters as in Fig. 3 with $\tau_{0}=10 \mu$ sec and $\omega \tau \ll 1$.

and (2). The situation is less clear regarding structuration effects in the damping constant [8-19]. Depending on the system parameters, some studies reported oscillations as a function of the film thickness, others didn't. Moreover, experiments that did find oscillations, do not agree whether the damping maxima occur at the maxima or the minima of the stiffness. The present work was largely motivated by this latter question.

According to (16) and (17), elastic and viscous forces show opposite behavior: The maxima of the stiffness correspond to minima of the damping function and vice versa. These oscillations are related to fluctuations of the number of molecular layers; the fluctuation strength $\delta f^{2}$ is largest close at half-integer filling, and almost vanishes at optimal filling. Since fluctuations weaken the stiffness and enhance dissipation, these quantities show op- posite behavior as a function of the film thickness. These features agree with recent experimental findings on an OMTCS film confined by a AFM tip of radius $R=15$ $\mathrm{nm}[18]$.

So far our discussion relied on the relaxation time approximation with constant $\tau$. One should keep in mind that, due to the broad many-body relaxation spectrum, the quantity $\tau$ in general depends on frequency. More importantly, the relaxation time varies with the film thickness and is largest in the ordered state. This means that in Eq. (17) the factors $\delta f^{2}$ and $\tau$ show an opposite oscillatory behavior. If the modulation of the relaxation time dominates, maxima of the damping coefficient occur in the ordered state where $L / \tilde{a}$ is an integer. As an illustration, we compare in Fig. 4 the damping coefficient for constant $\tau$ with that obtained from an Arrhenius type relaxation time $\tau=\tau_{0} e^{E_{L} / k_{B} T}$, with a modulated activation energy $E_{L}=E_{0}[1+\cos (2 \pi L / \tilde{a})] \tilde{a} / L$. For $E_{0}=0$ the damping maxima occur at intermediate states, whereas a high enough activation energy shifts them to optimal filling where $L \approx n \tilde{a}$. Such a change of the relaxation behavior could be related to the size and surface roughness of the AFM tip as discussed in [19], or to "jamming" at higher drift velocity of tip [13].

We conclude with a remark on the formal equivalence of the response function (15) to Maxwell's relaxation model for viscoelastic fluids. The present work adds two features that are essential for confined films: First, the modulation of the prefactor $\delta f^{2}$ is at the orign of the oscillatory behavior of both elastic and dissipative response. Second, the response function contains, beyond the Maxwell relaxation term $\phi$, a static contribution $K_{0}$ which completely changes the elastic behavior with respect to a Maxwell fluid. In view of these findings, the viscoelastic response of molecular layers arise from a subtle interplay of elastic energy and fluctuations.

Helpful discussions with A. Maali are gratefully acknowledged.
[1] J.N. Israelachvili, Intermolecular and Surface Forces, Academic Press London (1992)

[2] R.G. Horn, J.N. Israelachvili, J. Chem. Phys. 75, 1400 (1981)

[3] H. Christenson, J. Chem. Phys. 78, 6906 (1983)

[4] J.N. Israelachvili, R.M. Pashley, Nature 306, 249 (1983)

[5] S.J. O'Shea, M.E. Welland, J.B. Pethica, Chem. Phys. Lett. 223, 336 (1994)

[6] R. Lim, S.J. O'Shea, Phys. Rev. Lett. 88, 246101 (2003)

[7] J. Gao, W.D. Luedtke, U. Landman, Phys. Rev. Lett. 79, 705 (1997)

[8] S.J. O'Shea, M.E. Welland, Langmuir 14, 4186 (1998)

[9] S.P. Jarvis et al., J. Phys. Chem. B 104, 6091 (2000)

[10] M. Antognozzi, A.D.L. Humphris, M.J. Miles, Appl. Phys. Lett. 78, 300 (2001)

[11] S. Jeffrey et al., Phys. Rev. B 70, 054114 (2004)

[12] T. Uchihashi et al., Nanotechnology 16, S49 (2005)
[13] S. Patil et al., Langmuir 22, 6488 (2006)

[14] A. Maali et al., Phys. Rev. Lett. 96, 086105 (2006)

[15] S.J. O'Shea, Phys. Rev. Lett. 97, 179601 (2006)

[16] G.B. Kaggwa et al., Appl. Phys. Lett. 93, 011909 (2008)

[17] W. Hofbauer et al., Phys. Rev. E 80, 134104 (2009)

[18] S. de Beer, D. van den Ende, F. Mugele, Nanotechnology 21, 325703 (2010)

[19] S. de Beer, D. van den Ende, F. Mugele, J. Phys. Cond. Matt. 23, 112206 (2011)

[20] R. Kubo, M. Toda, N. Hashisume, Statistical Physics II, Non-equilibrium Statistical Mechanics, Springer Heidelberg (1985)

[21] C. Kittel, Introduction to Solid State Physics, Wiley New York (1996)

[22] M. Schmidt, H. Löwen, Phys. Rev. Lett. 76, 4552 (1996)

[23] R. Zangi, S.A. Rice, Phys. Rev. E 61, 660 (2000)

[24] C. Ghatak, K.G. Ayappa, Phys. Rev. E 64, 051507 
(2001)

[25] A. Vishnyakov, A.W. Neimark, J. Chem. Phys. 118, 7585 (2003)

[26] E. Kierlik, M.L. Rosinberg, Phys. Rev. A 44, 5025 (1991)

[27] A.L. Demirel, S. Granick, Phys. Rev. Lett. 77, 2261
(1996)

[28] T. Becker, F. Mugele, Phys. Rev. Lett. 91, 166104 (2003)

[29] L. Bureau, A. Arvengas, Phys. Rev. E 78, 061501 (2008)

[30] L. Bureau, Phys. Rev. Lett. 104, 218302 (2010) 